\title{
Effect of mental stress on cold pain in chronic tension-type headache sufferers
}

\author{
Stuart Cathcart · Anthony H. Winefield • \\ Kurt Lushington · Paul Rolan
}

Received: 18 March 2009/Accepted: 9 May 2009/Published online: 5 June 2009

(C) Springer-Verlag 2009

\begin{abstract}
Mental stress is a noted contributing factor in chronic tension-type headache $(\mathrm{CTH})$, however the mechanisms underlying this are not clearly understood. One proposition is that stress aggravates already increased pain sensitivity in CTH sufferers. This hypothesis could be partially tested by examining effects of mental stress on threshold and supra-threshold experimental pain processing in CTH sufferers. Such studies have not been reported to date. The present study measured pain detection and tolerance thresholds and ratings of supra-threshold pain stimulation from cold pressor test in CTH sufferers (CTH-S) and healthy Control (CNT) subjects exposed to a 60-min stressful mental task, and in CTH sufferers exposed to a 60-min neutral condition (CTH-N). Headache sufferers had lower pain tolerance thresholds and increased pain intensity ratings compared to controls. Pain detection and tolerance thresholds decreased and pain intensity ratings increased during the stress task, with a greater reduction in pain detection threshold and increase in pain intensity ratings in the CTH-S compared to CNT group. The results support the hypothesis that mental stress contributes to
\end{abstract}

S. Cathcart $(\square) \cdot$ A. H. Winefield

School of Psychology, Centre for Applied Psychological

Research, University of South Australia, Adelaide,

SA 5000, Australia

e-mail: Stuart.Cathcart@postgrads.unisa.edu.au;

stuartcathcart@hotmail.com

\section{K. Lushington}

Sleep Research Centre, University of South Australia,

Adelaide, SA, Australia

P. Rolan

Discipline of Pharmacology, School of Medical Sciences,

University of Adelaide, Adelaide, SA, Australia
CTH through aggravating already increased pain sensitivity in CTH sufferers.

Keywords Tension-type headache $\cdot$ Stress .

Pain sensitivity $\cdot$ Cold pressor

\section{Introduction}

Mental stress is noted as a contributing factor to chronic tension-type headache $(\mathrm{CTH})$, however the mechanisms underlying this are not clearly understood. One proposition is that stress aggravates already increased pain sensitivity in CTH sufferers $[1,2]$. Supporting this are findings that CTH sufferers are more susceptible to hyperalgesic effects of mental stress on cephalic pressure pain detection thresholds [3], and findings that mental stress-induced headache is associated with reduced pressure pain detection thresholds in CTH sufferers [4]. Pressure pain detection thresholds provide limited information on pain processing. Supra-threshold measures, such as pain tolerance and ratings of tonic pain intensity, provide additional information on the pain system, and may be more representative of clinical pain $[5,6]$. To date however, no studies have examined effects of mental stress on quantitative ratings of experimental pain tolerance or intensity in $\mathrm{CTH}$ sufferers.

Previous studies examining effect of mental stress on pain thresholds in CTH sufferers have used mechanical algometer as the pain stimulus [3, 4]. Such method is subject to multiple factors potentially impairing the reliability and validity of the results, such as algometer application rate, potential variability in the precise location of the stimulator, algometer stimulator dimensions, and experimenter factors (e.g. training in the technique). The cold pressor test can address these limitations. Particularly, 
cold pressor can be used to assess threshold and suprathreshold pain ratings, can control for stimulation intensity and application rate, is not subject to small variations in stimulation location, and is not as dependent on experimenter expertise to administer. Sensitivity to cold pressor has been found increased in headache sufferers in some studies [7, 8], but not others [9]. To date however, no studies have examined effects of mental stress on cold pressor in CTH sufferers. In healthy subjects, mental stress has been shown to increase sensitivity to cold pressor in some studies [10, 11], but not others [12, 13].

The present study measured pain detection and tolerance thresholds and ratings of supra-threshold pain stimulation from cold pressor test in CTH sufferers (CTH-S) and healthy control (CNT) subjects exposed to a 60-min stressful mental task, and in CTH sufferers exposed to a 60min neutral condition (CTH-N). The aim was to examine effects of mental stress on threshold and supra-threshold cold pain sensitivity in CTH sufferers. Socio-demographic and clinical data for the present sample have been reported elsewhere [14].

\section{Methods}

\section{Subjects}

Subjects were recruited via advertisements in local media and University of South Australia media requesting volunteers for a study on headaches. Written consent from each subject for study participation was obtained and the study was approved by the University's Human Research Ethics Committee. Potential volunteers underwent a diagnostic interview based on the International Classification of Headache Disorders (ICHD-II) criteria [15]. Inclusion criteria for the CTH group were: satisfying ICHD-II criteria for CTH, aged 18-65 years, not currently receiving (or having received in the last 12 months) intervention for headache, no psychiatric or major medical condition currently or in the last 12 months, no concurrent headache or pain symptoms or diagnoses (other than $\mathrm{CTH}$ ). Additionally, CTH subjects were required not to be taking, or not have taken in the last 3 months, regular analgesic medication other than $\leq 1,000 \mathrm{mg}$ daily of aspirin or paracetamol. All subjects were required to have not taken any analgesic on the day of examination, and were required to be headache free at presentation for the experimental session. Inclusion in the Control group required additional criteria of no past or current chronic pain or headache diagnoses, fewer than five headaches in the last year, and none within the last 6 months. Additionally, Control subjects were recruited following headache subjects to allow matching for age and gender. All recruited subjects completed the study procedures.

\section{Procedures}

The protocol involved exposing subjects to either an hourlong stressful mental task or an hour-long neutral condition, and measuring cold pressor responses before, during (30 min into the task), after task/neutral condition exposure. Headache subjects were randomly assigned to either the stress (CTH-S) or neutral (CTH-N) conditions, while all non-headache subjects (CNT) were exposed to the stress condition only, as we have previously shown that the same neutral condition does not effect changes in pain sensitivity in healthy subjects [16]. The sample contained $N=25$ CTH-S, $N=23$ CTH-N, and $N=23$ CNT subjects. All sessions were conducted in an interview room at the School of Psychology, University of South Australia, between 9.00 a.m. and 5.00 p.m. on Monday to Friday. The room was a constant $23^{\circ} \mathrm{C}$.

\section{Self-report questionnaires}

Prior to the experimental condition, subjects completed an in-house socio-demographic questionnaire and clinical interview detailed elsewhere [17]. Subjects also completed the State-Trait Anxiety Inventory [18], and the Centre for Epidemiological Studies-Depression Scale [19], due to possible effects of anxiety and depression on pain sensitivity.

\section{Mental stress task}

The stress task was adapted from one previously demonstrated to induce mental stress and headache [20], and involved subjects solving anagrams and arithmetic problems, presented via computer monitor. Anagrams had three levels of difficulty: 'Easy' anagrams were words of 8-10 letters with two sets of adjacent letters presented in reverse order. 'Difficult' anagrams were long words with letters presented in random order, and 'Insoluble' anagrams were 9- to 11-letter words with letters presented in random order and one letter omitted so no solution existed. Previous research has shown subjects can solve most of the easy anagrams but few of the difficult anagrams [20]. The arithmetic problems have been used in previous research by our group [3], and involve subtraction or addition of two-digit (e.g. 73-58) and three-digit (e.g. 576-283) problems. There was an equal amount of anagrams and arithmetic problems in each block, which were presented in random order.

The task involved presentation of each problem for $10 \mathrm{~s}$ on the monitor, during which subjects were required to solve the problem in their head (without the use of paper or pencil). Following the problem presentation screen, a screen with the words 'inter-trial interval' was presented 
for $5 \mathrm{~s}$, during which subjects verbalized their answer to the previous problem, or said 'pass' if they did not know the answer. The experimenter remained in the room with the subject for the entire time and pretended to enter their responses into a computer.

The task was divided into 12 'blocks' of 10 problems. Subjects were told they would receive performance feedback via the screen after each block, advising of their accuracy relative to previous participants, in the form of 'below average', 'average', or 'above average'. In fact, feedback was not based on performance, with subjects receiving 'below average' for eight blocks, and 'average' for four blocks. Subjects were also advised that the duration of the task was based on their performance, with the shortest possible duration being $30 \mathrm{~min}$ (if they got all problems correct), and the longest duration $1.5 \mathrm{~h}$ (if they got all of the problems incorrect). In fact, the task lasted for $1 \mathrm{~h}$, regardless of performance.

\section{Neutral condition}

The neutral condition involved subjects remaining seated in the interview room and browsing supplied magazines and newspapers for $60 \mathrm{~min}$. The experimenter remained in the room working on a computer, and did not engage with the subjects during the task.

\section{Cold pressor task}

The cold pressor task involved subjects immersing their right hand up to the wrist in a circulating water bath containing water maintained at $0^{\circ} \mathrm{C}$. Following immersion, subjects reported when the stimulation first became painful (pain detection threshold), then provided a rating of pain intensity every $10 \mathrm{~s}$ according to a visual analogue scale presented on a wall in front of them ranging from ' 0 - no pain' to ' 10 - extremely painful'. Subjects were requested to keep their hand in the water for a long as possible, and to withdraw their hand when they could no longer tolerate the pain. The experimenter recorded the pain detection and tolerance times using a stopwatch, which was also used by the experimenter to prompt subjects for tonic pain ratings every $10 \mathrm{~s}$ during hand immersion.

\section{Statistical methods}

Statistical analyses were conducted using the Statistical Package for the Social Sciences [21]. A two within [task (pre, during, post); cold pressor ratings (threshold, tolerance, rating)] and one between (CTH-S, CTH-N, CNT) factor repeated measures analysis of variance (RMANOVA) was used to explore group differences and effects of stress on cold pressor thresholds and pain ratings. Due to the majority of CTH sufferers reaching tolerance within $30 \mathrm{~s}$ of immersion, only pain ratings at 10 and $20 \mathrm{~s}$ were included in the tonic pain rating analyses, conducted thus, on $N=15$ CTH-S, $N=13$ CTH-N, and $N=13 \mathrm{CNT}$ subjects. Significant RMANOVA results were followed up with examination of simple main effects between each pair of groups (CTH-S vs CTH-N, CTH-S vs CNT, CTH-N vs CNT). ANOVA and Chi-square test were used to compare groups on socio-demographic and clinical data. Finally, split-file Pearson's $r$ correlations were conducted to examine relationships between experimental (cold pressor) pain, clinical characteristics (headache history, average headache severity, headache frequency over the last month), anxiety, and depression.

\section{Results}

Detailed analyses of socio-demographic and clinical data have been reported elsewhere [14] and hence are not reproduced herein. Briefly however, the total sample was comprised of $N=25$ males and $N=46$ females, with a mean age of 27.1 years (SD 7.4). There were no significant differences between groups on measures of age, depression, anxiety, or gender (all, $P>0.10$ ). There were no differences between the two headache groups (CTH-S, CTH-N) on measures of headache frequency, years of headache, or mean headache intensity (all $P>0.10$ ).

Figure 1 shows pain detection thresholds before, during and after task/neutral condition exposure in CTH-S, CTH-N, and CNT groups. Analyses showed a significant task effect $F(2,67)=6.76, P<0.01$, and a significant

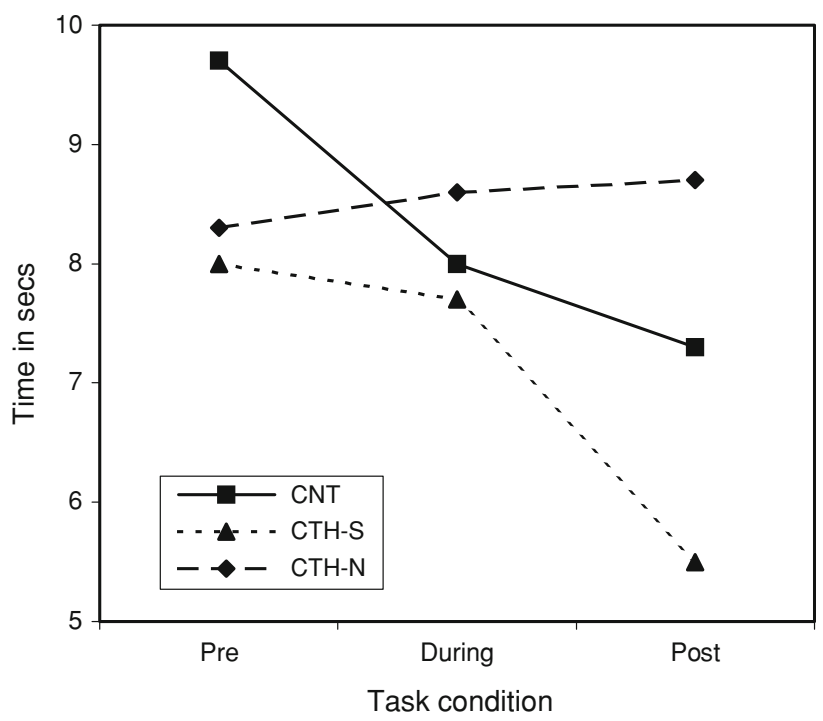

Fig. 1 Pain detection thresholds in headache subjects (CTH-S) and Controls (CNT) exposed to stress and headache subjects exposed to neutral task (CTH-N) 


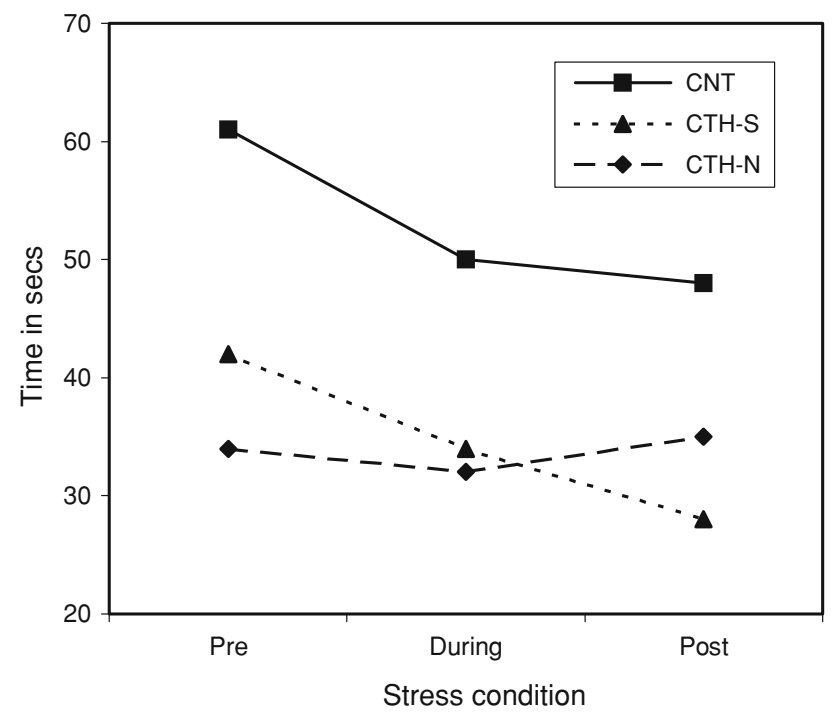

Fig. 2 Pain tolerance thresholds in headache subjects (CTH-S) and healthy Controls (CNT) exposed to stress and headache subjects exposed to neutral task (CTH-N)

task $\times$ group interaction $F(4,136)=3.93, \quad P<0.01$. Overall group effects were not significant $F(1,68)=0.18$, $P>0.10$. Analyses of simple main effects showed a greater decrease in pain detection thresholds during task in the CTH-S group compared with both the CNT group $F(1,46)=4.43, P<0.05$, and the CTH-N group $F(1,46)=$ $13.98, P<0.01$.

Figure 2 shows the pain tolerance thresholds before, during and after task/neutral condition exposure in CTH-S, CTH-N, and CNT groups. Analyses showed a significant group effect $F(2,68)=3.16, P<0.05$, a significant task effect $F(2,67) 13.54, P<0.01$, and a significant task $\times$ group interaction $F(4,136)=4.72, P<0.01$. Analyses of simple main effects showed pain tolerances were higher in the CNT group compared to both the CTH-S group $F(1,46)=3.77, P<0.05$, and the CTH-N group $F(1,46)=4.10, P<0.05$. There was a greater decrease in pain tolerance during task in the CTH-S group compared to the CTH-N group $F(1,44)=27.03, P<0.01$, and a greater decrease in the CNT compared to CTH-N group $F(1,46)=11.23, P<0.01$.

Figure 3 shows pain intensity ratings at 10 and $20 \mathrm{~s}$ after cold water immersion, before, during, and after task in the CTH-S, CTH-N, and CNT groups. Analyses showed a significant time effect $(10-20 \mathrm{~s}) F(1,38)=207.2, P<0.01$, a significant task effect $F(1,38)=4.56, P<0.05$, and a significant task $\times$ group interaction $F(2,38)=5.05, P=0.01$. Overall group effects approached significance $F(2,28)=$ $2.97, P=0.05$. Analyses of simple main effects showed pain ratings increased during the task in the CTH-S group compared to both the CNT group $F(1,26)=4.13, P<0.05$, and the CTH-N group $F(1,24)=13.15, P<0.01$, while overall

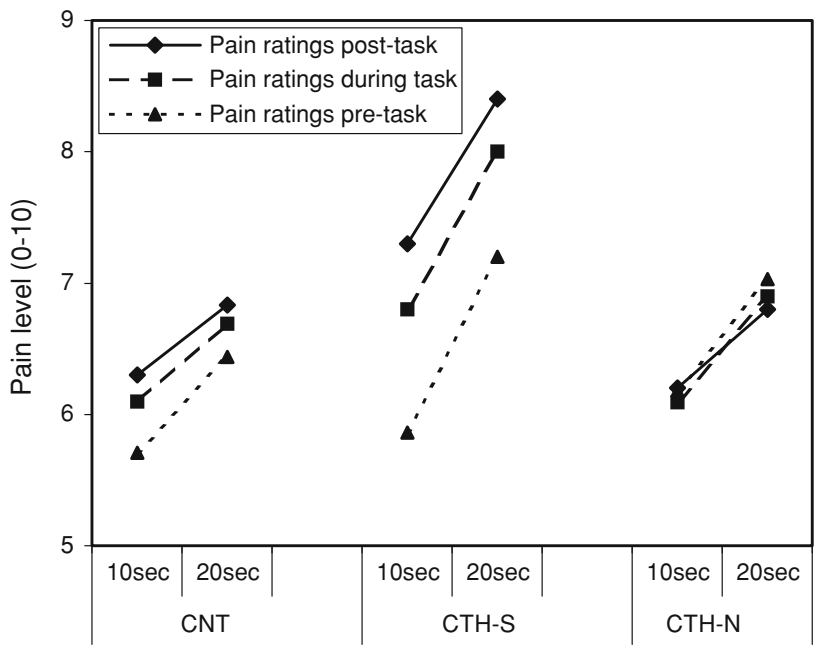

Fig. 3 Pain ratings in headache subjects (CTH-S) and healthy Controls (CNT) exposed to stress and headache subjects exposed to neutral task (CTH-N)

pain ratings were higher in the $\mathrm{CTH}-\mathrm{S}$ compared to the $\mathrm{CNT}$ group $F(1,26)=6.03, P<0.05$.

Correlation analyses revealed no pattern of significant relationships between cold pressor responses and psychological or clinical measures, except for pain intensity rating at $20 \mathrm{~s}$ during and post-task, which were both positively correlated with headache frequency over the last month (all $r>0.40, P<0.05)$.

\section{Discussion}

Group differences in pain sensitivity

The present results indicate reduced tolerance but not detection thresholds to cold induced pain at the hand in CTH sufferers. Such results are consistent with suggestions of central sensitization and an increased general pain sensitivity in CTH sufferers $[1,22,23]$. The results for detection thresholds are consistent with the only previous study to examine cold pressor exclusively in CTH sufferers [9], and are consistent with Marlowe [24], who reported decreased tolerance but not detection thresholds to ice placed at the temple in frequent tension-type headache sufferers. Similarly, differences between CTH and Control subjects on heat pain thresholds have been less often observed than have differences in pressure pain thresholds, particularly in CTH subjects from the general population, as assessed in the current study. It may be that such subjects represent less severe cases of CTH, with accordingly less sensitization [1]. In contrast to the present results, Bishop et al. [9] failed to find reduced tolerance to cold pressor in CTH subjects. The conflicting results may be due 
to method: Bishop et al's [9] cold pressor used un-circulating water at $0-2^{\circ} \mathrm{C}$, while we used circulating water at $0^{\circ} \mathrm{C}$. Circulating water avoids heat build up around the hand [25], presenting a more painful (and possibly less tolerable) stimulus.

The present study found increased pain intensity ratings that approached significance $(P=0.05)$ in CTH sufferers compared to CNT. The result extends previous findings of increased sensitivity to cold pressor in migraine and mixed headache diagnostic groups [7, 8]. Increased pericranial muscle tenderness is a common finding in CTH sufferers [17, 26, 27], indicating sensitivity to supra-threshold mechanical stimulation is increased in pericranial region of CTH sufferers. The present results suggest CTH sufferers also have increased sensitivity to supra-threshold cold pain stimulation at extra-cephalic locations.

Ashina et al. [23] suggest supra-threshold testing may be more sensitive than pain detection thresholds for assessing central sensitization in $\mathrm{CTH}$. The present findings of increased supra-threshold pain rating and decreased pain tolerance but not detection thresholds in CTH sufferers supports this suggestion. Bendsten et al. [28] found qualitatively altered stimulus-response function to suprathreshold pressure pain stimulation in $\mathrm{CTH}$ sufferers. The present results found no group differences in pain increase from 10 to $20 \mathrm{~s}$ during cold pressor immersion. This indicates that although overall rating of supra-threshold cold stimulation is increased in CTH sufferers, the stimulus-response function is not altered. However, a conclusion cannot be made due to the limited number of stimulusresponse data points in the present study. Further research using more data points is needed to test this hypothesis.

\section{Effects of stress on pain sensitivity}

Olesen [29] and others [1, 30] have suggested mental stress may contribute to $\mathrm{CTH}$ through aggravating already increased pain sensitivity in CTH sufferers. The present results support such a hypothesis, indicating mental stress reduces pain detection threshold and increases supra-threshold pain ratings more in CTH sufferers than healthy Controls.

A previous study found a brief (15 min) mental stress task decreased pericranial pressure pain thresholds but not cold pain thresholds more in CTH sufferers than in healthy controls [3]. In contrast, the present study found mental stress decreased cold pain detection thresholds more in CTH sufferers than controls. The difference may be due to the longer stress task used in the present study, or the use of cold pressor in the present study compared to an ice cube held against the wrist as the cold pain stimulus in the previous study [3].

The present study also extended previous findings by examining, for the first time, effects of induced mental stress on supra-threshold cold pain ratings in CTH sufferers. The results indicated that stress increased overall pain intensity from cold pressor more in the headache sufferers than in the control group. Further, the results indicated no difference in supra-threshold pain ratings between the healthy Controls exposed to the stress task and the headache group exposed to the neutral condition. This suggests that group differences in supra-threshold pain ratings may be due to effects of the mental task in the headache group. In contrast, mental stress did not affect the rate of pain increase (from 10 to $20 \mathrm{~s}$ ) during cold pressor immersion in headache or control subjects, suggesting stress does not alter the stimulus-response function to cold induced pain. Mental stress-induced analgesia typically involves a rightward shift in the stimulusresponse function of central nociceptive neurons [31], resulting in an increased threshold and decreased intensity with no alteration in the shape of the response function. It may be that hyperalgesic effects of mental stress, as observed in the present study, conversely involve a leftward shift in the response function. The present finding that stress reduced threshold and increased intensity, without altering rate of increase, is consistent with this suggestion. Again, a conclusion cannot be made due to the limited number of stimulus-response data points.

It is interesting to note that the present analyses found a group difference on pain tolerance but not detection threshold, and a group $\times$ task effect on pain detection but not tolerance threshold. This result suggests enhanced effects of mental stress on pain processing in CTH sufferers may partially operate through different pain mechanisms than those underlying increased pain sensitivity at rest in CTH sufferers. For example, increased baseline pain sensitivity in CTH sufferers may be due to sensitization (indexed preferentially in reduced tolerance and increased supra-threshold response), while stress may aggravate the increased pain sensitivity through reducing threshold to noxious input from the periphery, and increasing supra-threshold response. Further research to examine this hypothesis may aid in elucidating central mechanisms of stress-induced headache.

Relationship of experimental pain to clinical pain and psychological measures

The relevance of experimental pain sensitivity to clinical pain has been widely debated [6, 32]. As chronic pain involves tonic sensation above threshold, tonic suprathreshold pain may be more relevant than pain thresholds to clinical pain [6]. Consistent with this, we found no correlation between pain thresholds and headache activity or psychological measures, but did find a correlation between pain intensity ratings and headache activity over the last month in the headache group. Previous studies report increased muscle tenderness is related to headache 
activity in CTH sufferers [17, 33, 34], indicating increased pain response in pericranial myofascia is of clinical relevance in CTH. The present results suggest a generalized increase in pain response may also be of clinical relevance to CTH. Conclusions are tentative however due to the lack of a consistent pattern of correlations across cold pressor and clinical pain measures in the present study.

\section{Limitations to the present study}

A number of limitations to the present study warrant mention. We only used subjective measures of stress. However, the majority of research indicates that where differences between headache and Control subjects have been found, they have generally been found on subjective but not physiological indices of stress [35]. Similarly, we used self-report pain measures. However, self-report represents maximal integration of the stress and pain systems, and most previous research has used self-report to demonstrate increased pain sensitivity in CTH sufferers (e.g. [22, 25, 27]), and hyperalgesic effects of stress on pain sensitivity in healthy humans (e.g. $[10,11])$. Indeed, previous research found effects of stress on pain report but not nociceptive reflex [36, 37]. Finally, we did not examine for possible gender effects in our study due to the sample size being too small for reliable analysis. However, as groups did not differ on measures of gender, such effects could not account for the observed group differences in pain sensitivity. Additionally, the issue of gender effects on pain sensitivity is at present unclear: Some authors have reported gender effects on pain sensitivity [38, $39]$, while others have not [40, 41]. Further research examining possible gender differences in the present protocol are required to address this issue.

\section{Conclusions}

The present study found reduced cold pain tolerance thresholds and increased rating of supra-threshold cold pain in CTH sufferers compared to healthy controls. Further, the present study found mental stress reduced pain threshold and increased supra-threshold pain ratings more in CTH sufferers than healthy Controls. Taken together, the results support the hypothesis that mental stress contributes to $\mathrm{CTH}$ through aggravating already increased pain sensitivity in CTH sufferers.

\section{Conflict of interest None}

\section{References}

1. Bendsten L (2000) Central sensitization in tension-type headache-possible pathophysiological mechanisms. Cephalalgia 20:486-508. doi:10.1046/j.1468-2982.2000.00070.x
2. Nash JM, Thebarge RW (2006) Understanding psychological stress, its biological processes, and impact on primary headache. Headache 46:1377-1386

3. Cathcart S, Petkov J, Pritchard D (2008) Effects of induced stress on experimental pain sensitivity in chronic tension-type headache sufferers. Eur J Neurol 15:552-558. doi:10.1111/j.1468-1331. 2008.02124.x

4. Janke EA, Holroyd KA, Romanek K (2004) Depression increases onset of tension-type headache following laboratory stress. Pain 111:230-238. doi:10.1016/j.pain.2004.06.007

5. Kim H, Neubert JK, Rowan JS, Brahim JS, Iadarola MJ, Dionne RA (2004) Comparison of experimental and acute clinical pain response in humans as pain phenotypes. J Pain 5(7):377-384. doi: 10.1016/j.jpain.2004.06.003

6. Edwards RR, Sarlani E, Wesselmann U, Fillingim RB (2005) Quantitative assessment of experimental pain perception: multiple domains of clinical relevance. Pain 114:315-319. doi:10.1016/ j.pain.2005.01.007

7. Ukestad LK, Wittrock DA (1996) Pain perception and coping in female headache sufferers and headache-free controls. Health Psychol 15(1):65-68. doi:10.1037/0278-6133.15.1.65

8. Hassinger HJ, Semenchuk EM, O'Brien WH (1999) Appraisal and coping response to pain and stress in migraine headache sufferers. J Behav Med 22(4):327-340. doi:10.1023/A: 1018722002393

9. Bishop KL, Holm JE, Borowiak DM, Wilson BA (2001) Perception of pain in women with headache: a laboratory investigation of the influence of pain-related anxiety and fear. Headache 41:494-499. doi:10.1046/j.1526-4610.2001.01087.x

10. Zelman DC, Howland EW, Nichols SN, Cleeland CS (1991) The effects of induced mood on laboratory pain. Pain 46:105-111. doi:10.1016/0304-3959(91)90040-5

11. Caceres C, Burns JW (1997) Cardiovascular reactivity to psychological stress may enhance subsequent pain sensitivity. Pain 69:237-244. doi:10.1016/S0304-3959(96)03289-7

12. Absi M, Petersen KL (2003) Blood pressure but not cortisol mediates stress effects on subsequent pain perception in healthy men and women. Pain 106:285-295. doi:10.1016/S0304-3959 (03)00300-2

13. Girdler SS, Maixner W, Naftel HA, Stewart PW, Moretz RL, Light KC (2005) Cigarette smoking, stress-induced analgesia and pain perception in men and women. Pain 114:372-385. doi: 10.1016/j.pain.2004.12.035

14. Cathcart S, Petkov J, Winefield AH, Lushington K, Rolan P (2009) Central mechanisms of stress-induced headache. Cephalalgia (in press)

15. International Headache Society (2004) The international classification of headache disorders, 2nd edn. Cephalalgia 24(Suppl 1):9-160. doi:10.1111/j.1468-2982.2003.00824.x

16. Cathcart S, Winefield AH, Lushington K, Rolan P (2009) Reliability of temporal summation and diffuse noxious inhibitory controls. Headache (submitted)

17. Cathcart S, Pritchard D (2008) Daily hassles and pain sensitivity in chronic tension-type headache sufferers. Stress Health 24:123127. doi: $10.1002 / \mathrm{smi} .1167$

18. Spielberger CD, Gorsuch RL, Lushene RL (1970) Manual for the state trait anxiety inventory. Consulting Psychologists Press, Palo Alto

19. Radloff LS (1977) The CES-D scale: a self-report depression scale for research in the general population. Appl Psychol Meas 1:385-481. doi:10.1177/014662167700100306

20. Martin PR, Todd J, Reece J (2005) Effects of noise and a stressor on head pain. Headache 45:1353-1364

21. Inc SPSS (2007) Statistical package for the social sciences, release 15.0. Vesta Services Inc., Chicago

22. Ashina A, Babenko L, Jensen R, Ashina M, Mageri W, Bendsten L (2005) Increased muscular and cutaneous pain sensitivity in 
cephalic region in patients with chronic tension-type headache. Eur J Neurol 12(7):543-549. doi:10.1111/j.1468-1331.2005. 01023.x

23. Ashina S, Bendtsen L, Ashina M, Magerl W, Jensen R (2006) Generalized hyperalgesia in patients with chronic tension-type headache. Cephalalgia 26:940-948. doi:10.1111/j.1468-2982. 2006.01150.x

24. Marlowe N (1991) Pain sensitivity and headache: an examination of the central theory. J Psychosom Res 36:17-34. doi:10.1016/ 0022-3999(92)90110-N

25. Mitchell LA, MacDonald RA, Brodie EE (2004) Temperature and the cold pressor test. J Pain 5(4):233-238. doi:10.1016/j. jpain.2004.03.004

26. Langemark M, Olesen J (1987) Pericranial tenderness in tension headache. Cephalalgia 7:249-255. doi:10.1046/j.1468-2982.1987. 0704249.x

27. Jensen R, Bendtsen L, Olesen J (1998) Muscular factors are of importance in tension-type headache. Headache 38:10-17. doi: 10.1046/j.1526-4610.1998.3801010.x

28. Bendsten L, Jensen R, Olesen J (1996) Qualitatively altered nociception in chronic myofascial pain. Pain 65:259-264. doi: 10.1016/0304-3959(95)00239-1

29. Olesen J (1991) Clinical and pathophysiological observations in migraine and tension-type headache explained by integration of vascular, supra-spinal and myofascial inputs. Pain 46:125-132. doi:10.1016/0304-3959(91)90066-7

30. Fumal A, Schoenen J (2008) Tension-type headache: current research and clinical management. Lancet Neurol 7:70-83. doi: 10.1016/S1474-4422(07)70325-3

31. Gebhart GF (2004) Descending modulation of pain. Neurosci Biobehav Rev 27:729-737. doi:10.1016/j.neubiorev.2003.11.008

32. Gracely R (1999) Studies of pain in human subjects. In: Wall P, Melzack R (eds) Textbook of pain. Churchill Livingstone, New York, pp 385-407
33. Jensen R, Rasmussen BK, Pederson B, Olesen J (1993) Muscle tenderness and pressure pain thresholds in headache: a population study. Pain 52:193-199. doi:10.1016/0304-3959(93)90131-8

34. Buchgreitz L, Lyngberg AC, Bendtsen L, Jensen R (2006) Frequency of headache is related to sensitization: a population study. Pain 123:19-27. doi:10.1016/j.pain.2006.01.040

35. Wittrock DA, Myers TC (1998) The comparison of individuals with recurrent tension-type headache and headache free controls in physiological response, appraisal, and coping with stressors: a review of the literature. Ann Behav Med 20(2):118-134. doi: 10.1007/BF02884458

36. French DJ, France CR, France JL, Arnott LF (2005) The influence of acute anxiety on assessment of nociceptive flexion reflex threshold in healthy young adults. Pain 114:358-363. doi: 10.1016/j.pain.2004.12.034

37. Koh CW, Drummond PD (2006) Dissociation between pain and the nociceptive blink reflex during psychological arousal. Clin Neurophysiol 117:851-854

38. Ge HY, Madeleine P, Arendt-Nielson L (2005) Gender differences in pain modulation evoked by repeated injections of glutamate into the human trapezius muscle. Pain 13:134-140. doi: 10.1016/j.pain.2004.09.041

39. Serrao M, Rossi P, Sandrini G, Parisi L, Amabile GA, Nappi G, Pierelli F (2004) Effects of noxious inhibitory controls on temporal summation of the RIII reflex in humans. Pain 112:353-360. doi:10.1016/j.pain.2004.09.018

40. Nie H, Arendt-Nielsen L, Anderson H, Graven-Nielsen T (2005) Temporal summation of pain evoked by mechanical stimulation in deep and superficial tissue. J Pain 6(6):348-355. doi:10.1016/ j.jpain.2005.01.352

41. Sandrini G, Rossi P, Milanov I, Serrao M, Cecchini AP, Nappi G (2006) Abnormal modulatory influence of diffuse noxious inhibitory controls in migraine and chronic tension-type headache patients. Cephalalgia 26:782-789. doi:10.1111/j.1468-2982.2006.01130.x 